\title{
Fever in the Returning International Traveller Initial Assessment Guidelines
}

\section{Preamble}

The Committee to Advise on Tropical Medicine and Travel (CATMAT) provides the Public Health Agency of Canada (PHAC) with ongoing and timely medical, scientific, and public health advice relating to tropical infectious diseases and health risks associated with international travel. PHAC acknowledges that the advice and recommendations set out in this statement are based upon the best current available scientific knowledge and medical practices, and is disseminating this document for information purposes to both travellers and the medical community caring for travellers.

Persons administering or using drugs, vaccines, or other products should also be aware of the contents of the product monograph(s) or other similarly approved standards or instructions for use. Recommendations for use and other information set out herein may differ from that set out in the product monograph(s) or other similarly approved standards or instructions for use by the licensed manufacturer(s). Manufacturers have sought approval and provided evidence as to the safety and efficacy of their products only when used in accordance with the product monographs or other similarly approved standards or instructions for use.

\section{Introduction}

Fever in the international traveller is a common syndrome seen in the post-travel setting, and may herald serious and life-threatening illness, the most important of which is malaria. All febrile patients or patients complaining of fever should therefore be asked about recent travel. While fever in the returning traveller may be due to benign self-limited infections, such as common agents of travellers' diarrhea, or typical cosmopolitan causes unrelated to travel, it must be initially construed as a medical emergency, and warrants prompt and thorough evaluation. Accurate diagnosis and appropriate management necessitate a comprehensive travel history, including details about travel itinerary (destination, arrival and departure dates), the style of travel, pre-travel preparations, as well as potential exposures or medical treatment or care received abroad and a complete physical examination, with particular attention paid to systems with localizing symptoms or signs. The judicious use of laboratory testing should follow, with minimal essential tests outlined in the stepwise algorithm below.

\footnotetext{
*Members: Dr. P.J. Plourde (Chair); Dr. C. Beallor; Dr. A. Boggild; Dr. J. Brophy; Dr. M. Crockett; Dr. W. Ghesquiere; Ms. A. Henteleff; Dr. A. McCarthy; Dr. K. L. McClean Ex-Officio Representatives: Dr. G. Brunette, Dr. J. Creaghan, Dr. P. Charlebois; Dr. M. Tepper; Dr. P. McDonald; Dr. J. Given Liaison Representatives: Dr. C. Greenaway; Dr. A. Pozgay; Dr. C. Hui; Dr. P. Teitelbaum

Member Emeritus: Dr. C. William L. Jeanes

Consultant: Dr. S. Schofield
}

† This Statement was prepared by Dr. A. Boggild, Dr. W. Ghesquiere, Dr. A. McCarthy and approved by CATMAT 
This document is designed to:

- Guide front-line clinicians, including those working in the emergency room, walk-in clinics, and primary care practices, in the initial evaluation and management of fever in the returning traveller;
- Provide front-line clinicians without ready access to inpatient, internal medicine, or infectious diseases support with guidance in the evaluation and management of fever in the returning traveller beyond the initial phase.

\section{Epidemiology of fever in the returning traveller}

Fever in the returning traveller may be due to tropical infections or illnesses that have more of a cosmopolitan distribution globally. Numerous large series of illness after international travel have repeatedly identified malaria (20$30 \%)^{1}$, acute travellers' diarrhea (10-20\%), and respiratory tract infections (10-15\%) as the top causes of fever in travellers $^{2-11}$ (Table 1 ). Other common causes of fever in the returning traveller include dengue (5\%), enteric fever due to Salmonella enterica serovar Typhi or Paratyphi (2-7\%), skin and soft tissue infections (2-11\%), rickettsioses (3\%), urinary tract and sexually transmitted infections (2-3\%), viral hepatitis (3\%), and non-specific viral or mononucleosislike syndrome (4-25\%) ${ }^{2-11}$. In febrile returning pediatric travellers, malaria (35\%), viral syndromes (28\%), unspecified febrile illnesses (11\%), dengue (6\%), and enteric fever (6\%) are the most well-represented etiologies ${ }^{12}$. Fever after international travel may be due to non-infectious causes as well, and include diverse etiologies such as drug reactions, pulmonary emboli, inflammatory conditions such as inflammatory bowel disease (IBD), malignancy, and hyperthyroidism.

Destination-specific analysis is helpful for establishing the epidemiology of travel-acquired illness ${ }^{13}$ (Table 2). A history of travel to the Indian sub-continent in a febrile returned traveller should raise the suspicion of enteric fever: of 416 cases of imported typhoid in the United Kingdom over a three year period, 70\% were imported from India and Pakistan ${ }^{14}$. Malaria, on the other hand, illustrates a very different epidemiology among imported cases, with relative risks highest among travellers in sub-Saharan Africa ${ }^{15}$. Unlike malaria, dengue fever is more likely to be acquired from South Asia, Southeast Asia and Latin America, rather than from Africa ${ }^{13}$.
Purpose of travel is another useful piece of the travel history. People who travel for the purpose of visiting friends and relatives (VFR), including VFR children, constitute a particular risk group for acquisition of travel-related infections ${ }^{12}$, particularly malaria ${ }^{16}$ and enteric fever ${ }^{4}$, and in the case of VFR children, acute viral hepatitis ${ }^{17}$.

A thorough travel history with an understanding of what illnesses are possible based on dates and style of travel, geographic risks, and specific travel itinerary including activities and behaviors is essential to narrowing the differential diagnosis and ruling out life-threatening travelacquired infections. Incubation period, defined as the time from initial exposure and infection to manifestation of symptoms, is also useful in refining the differential diagnosis. For instance, travel-acquired illnesses with short incubation periods including dengue, chikungunya, and travellers' diarrhea, can be reliably excluded if symptoms do not manifest until $>2$ weeks after leaving the endemic area. Similarly, travel-acquired illnesses with long incubation periods such as tuberculosis (TB), hepatitis B (HBV), or visceral leishmaniasis can be discounted as travel-related if symptoms occur within days of returning from a one-week trip abroad. It is important to consider the earliest and latest possible dates of exposure in ill returned short and long-term travellers to better inform the use of the incubation period in formulating a differential diagnosis. The following step-wise algorithm is designed to guide the clinician through his or her initial assessment of a returned traveller with fever, and in rare cases, to provide further guidance when inpatient support or specialist consultation is delayed or unavailable. 
Table 1: Top specific diagnoses in single $e^{2 ; 3 ; 5-11}$ and multi-centre ${ }^{4 ; 12}$ analyses of fever in the returning traveller

\begin{tabular}{|c|c|}
\hline 1. Malaria (20-30\%) & 6. Skin and soft tissue infections (2-11\%) \\
\hline 2. Acute travellers' diarrhea / gastroenteritis (10-20\%) & $\begin{array}{l}\text { 7. Rickettsioses, including African tick bite fever, } \\
\text { Mediterranean spotted fever (tick typhus) (3\%) }\end{array}$ \\
\hline 3. Respiratory tract infections (RTI) (10-15\%) & $\begin{array}{l}\text { 8. Acute urinary tract infection / sexually transmitted infection } \\
\text { (UTI / STI) (2-3\%) }\end{array}$ \\
\hline 4. Dengue fever ( $5 \%)$ & 9. Viral hepatitis (3\%) \\
\hline $\begin{array}{l}\text { 5. Enteric fever (S. enterica serovar Typhi or Paratyphi) } \\
(2-7 \%)\end{array}$ & 10. Mononucleosis- or viral-like syndrome (4-25\%) \\
\hline \multicolumn{2}{|c|}{ (CATMAT statements are available at http://www.phac-aspc.gc.ca/tmp-pmv/catmat-ccmtmv/index-eng.php) } \\
\hline Sub-Saharan Africa & Malaria, RTI, diarrheal illness, skin and soft tissue infections \\
\hline Southeast Asia & Dengue fever, RTI, diarrheal illness, malaria \\
\hline South Central Asia / Indian sub-continent & Enteric fever, dengue fever, malaria, diarrheal illness \\
\hline South America & Diarrheal illness, RTI, dengue fever, malaria \\
\hline Caribbean / Central America & Diarrheal illness, RTI, dengue fever, malaria \\
\hline
\end{tabular}




\title{
Evaluation of fever in the returning traveller
}

\section{Objective: Exclusion of life-threatening, highly communicable, or treatable illness}

\author{
STEP 1: History of travel
}

\section{A. PRE-TRAVEL PREPARATIONS}

Pre-travel consultation: date, location, contact details

Immunizations received and dates; oral versus injectable formulation if applicable; completion of full vaccination series for travel and for routine childhood immunizations

Malaria prophylaxis: drug, dose, schedule, adherence, duration, side effects

Other prophylaxis: drug, dose, schedule, adherence, duration, side effects

Other personal protective measures: standby treatment of malaria / travellers' diarrhea; bednets; clothing; insecticide use

Air transportation preparations: deep vein thrombosis (DVT) prophylaxis, medications for jet lag

Environmental risk preparations: sun, extreme heat, altitude

Dates of travel (approximation of incubation period)

Season of travel: rainy, dry

Specific destinations: regions; urban, rural; proximity to fresh water; jungle, desert

Reason for travel: tourism, business, visiting friends and relatives (VFR), other

Style of travel: accommodation; off typical tourist routes; camping, trekking

Local population: possible TB contacts, outbreaks, illnesses

Transportation: crowding; use of animals such as camels, elephants 


\begin{tabular}{|c|c|}
\hline \multirow[t]{6}{*}{ C. EXPOSURE HISTORY* } & Street foods / Local water (enteric fever, travellers' diarrhea) \\
\hline & Arthropod bites (malaria, dengue, chikungunya, arboviruses, Rickettsia, African trypanosomiasis) \\
\hline & Uncooked meat / unpasteurized dairy (trichinosis, brucellosis, toxoplasmosis) \\
\hline & $\begin{array}{l}\text { Blood and body fluid exposure: sexual encounters, tattoos, piercings, injections including } \\
\text { immunizations, intravenous (IV) drug use, and rabies post-exposure prophylaxis (PEP) } \\
\text { (human immunodeficiency virus [HIV], HBV, hepatitis C virus [HCV], herpes simplex virus } \\
\text { [HSV], syphilis, gonorrhea (gonococcus) / Chlamydia trachomatis [GC/CT]) }\end{array}$ \\
\hline & Fresh water activities: swimming, kayaking, rafting (schistosomiasis, leptospirosis) \\
\hline & Safari (rickettsioses, African trypanosomiasis) \\
\hline
\end{tabular}

*For epidemiology of specific diseases, please refer to websites of the WHO (World Health Organization) (http://www.who.int/ topics/en/), or the Public Health Agency of Canada (PHAC) (http://www.phac-aspc.gc.ca/tmp-pmv/info/index-eng.php), the U.S. Centers for Disease Control and Prevention (CDC) (http://wwwnc.cdc.gov/travel/content/diseases.aspx). 
STEP 2: History of fever and clinical features

\begin{tabular}{|c|c|c|}
\hline \multirow[t]{9}{*}{ A. FEVER PATTERN } & \multirow[t]{3}{*}{ Daily / Continuous } & Common: malaria, travellers' diarrhea, RTI, enteric fever \\
\hline & & Uncommon: rickettsioses \\
\hline & & Rare: HIV, Q-fever, brucellosis, TB \\
\hline & \multirow[t]{3}{*}{ Saddleback (biphasic) } & Common: malaria, dengue \\
\hline & & Uncommon: leptospirosis \\
\hline & & Rare: yellow fever, arbovirus \\
\hline & \multirow[t]{3}{*}{ Relapsing } & Common: malaria, enteric fever \\
\hline & & Uncommon: amoebic liver abscess, leptospirosis \\
\hline & & Rare: HIV, Q-fever, brucellosis, TB, endemic mycoses, borreliosis \\
\hline \multirow{9}{*}{$\begin{array}{l}\text { B. INCUBATION PERIOD } \\
\text { (DEFINITION: TIME FROM } \\
\text { EXPOSURE / INFECTION TO } \\
\text { DISEASE MANIFESTATION) }\end{array}$} & \multirow[t]{3}{*}{$<2$ weeks } & Common: malaria, travellers' diarrhea, dengue, RTI, influenza \\
\hline & & Uncommon: rickettsioses, meningitis \\
\hline & & Rare: yellow fever, arbovirus, viral hemorrhagic fever \\
\hline & \multirow[t]{3}{*}{ 2-6 weeks } & Common: malaria, enteric fever \\
\hline & & $\begin{array}{l}\text { Uncommon: hepatitis A virus (HAV), katayama fever, } \\
\text { amoebic liver abscess, hepatitis E virus }\end{array}$ \\
\hline & & Rare: HIV, Q-fever, brucellosis, East African trypanosomiasis \\
\hline & \multirow[t]{3}{*}{$>6$ weeks } & Common: malaria \\
\hline & & Uncommon: HBV, amoebic liver abscess, TB \\
\hline & & $\begin{array}{l}\text { Rare: HIV, visceral leishmaniasis, endemic mycoses, } \\
\text { West African trypanosomiasis, HCV }\end{array}$ \\
\hline
\end{tabular}




\begin{tabular}{|c|c|c|}
\hline \multirow[t]{9}{*}{ C. FEVER DURATION AT VISIT } & \multirow[t]{3}{*}{$<7$ days } & Common: malaria, travellers' diarrhea, dengue, enteric fever, RTI \\
\hline & & Uncommon: rickettsioses, leptospirosis, meningitis \\
\hline & & Rare: yellow fever, arbovirus \\
\hline & \multirow[t]{3}{*}{ 7-21 days } & Common: malaria, enteric fever \\
\hline & & Uncommon: rickettsioses, viral hepatitis, leptospirosis \\
\hline & & Rare: HIV, Q-fever, brucellosis \\
\hline & \multirow[t]{3}{*}{$>21$ days } & Common: malaria, enteric fever \\
\hline & & Uncommon: TB, HBV, bacterial endocarditis \\
\hline & & Rare: HIV, Q-fever, brucellosis \\
\hline
\end{tabular}

Arbovirus: includes other mosquito-borne viruses such as chikungunya, mayaro, O'nyong-nyong, Ross River, sindbis, equine encephalitis, WNV, La Crosse, Oropouche, Rift Valley fever, and tick-borne viruses such as Kyasanur Forest, Omsk, and Crimean-Congo hemorrhagic fever 
STEP 3: Primary laboratory investigations after comprehensive physical examination including thorough skin examination

Returned travellers with fever should undergo at least the following investigations:

The febrile traveller to a malaria endemic area ${ }^{1}$ should be considered to have malaria until proven otherwise.

Travel history should be cited on all laboratory requisitions.

1. Complete blood count with differential; liver enzymes; electrolytes; creatinine

2. Malaria smears \pm antigen detection dipstick at least 3 times over $24-48$ hours

3. Blood cultures $x 2$ (S. enterica serovar Typhi or Paratyphi; meningococcus; common agents of bacteremia)

4. Urinalysis ( \pm urine culture)

\section{Consider the following supplementary tests based on history and epidemiology:}

5. Stool culture for enteropathogens x 1 (Salmonella, Shigella, Campylobacter, Yersinia, E. coli O157:H7)

6. Chest x-ray

7. Stool for ova and parasites (Cyclospora, Cryptosporidium, Entamoeba histolytica, Giardia)

8. Dengue serology if probable incubation period $<2$ weeks AND traveller to South Asia, Southeast Asia, or Latin America

9. Acute serology tube to be saved in lab and paired with convalescent sera if no diagnosis in 10-14 days

** Consider referral to a person with expertise in tropical medicine or infectious diseases (as available) if primary laboratory investigations yield no definitive diagnosis and patient is not improving, or patient is at risk of complications (e.g., pregnant women, children, underlying co-morbidities, immunocompromised, etc). Additional support, if required, may be sought through the Canadian Malaria Network (http://www.phac-aspc.gc.ca/tmp-pmv/quinine/index-eng.php)** 
STEP 4: Empiric management for presumed travellers' diarrhea or enteric fever \pm rickettsioses in adult travellers $\mathrm{i}$, ii

Consider if patient fulfills the following criteria:

1. Undifferentiated fever; and

2. Negative malaria smears ${ }^{1}$; and

3. Patient has not improved over past 48-72 hours; and

4. All other cultures and tests have been ordered and are pending or non-contributory

\begin{tabular}{l|l}
\hline Travelled to the Indian Sub-Continent or Southeast Asia & Travelled outside the Indian Sub-Continent or Southeast Asia \\
\hline $\begin{array}{l}\text { 1. Azithromycin } 1000 \mathrm{mg} \text { po once daily } \mathrm{x} 5 \text { days } \\
\begin{array}{l}\text { (alternative: } 1000 \mathrm{mg} \text { po once on day } 1 \text {, then } 500 \mathrm{mg} \\
\text { once daily } \mathrm{x} 6 \text { days } \pm\end{array}\end{array}$ & $\begin{array}{l}\text { 1. Ciprofloxacin } 500 \mathrm{mg} \text { po BID } \times 3-7^{*} \text { days } \pm \\
\text { 2. Doxycycline** } 100 \mathrm{mg} \text { po BID } 7 \text { days }\end{array}$ \\
\hline
\end{tabular}

i). Step 4 is not a substitute for Step 3 (Primary laboratory investigations after comprehensive physical examination), nor should empiric therapy preclude ongoing and close follow-up of the patient. Undifferentiated fever refers to fever without prominent localizing symptoms or signs, and is often the presenting complaint in many travel-acquired illnesses including malaria and enteric fever. It is essential that all febrile returned travellers to potentially malarious areas undergo malaria screening with 2-3 smears over 48 hours \pm rapid antigen testing. Adult patients who have not improved substantially, either subjectively or objectively, over the previous 48-72 hours are candidates for empiric therapy as above while investigations are pending. These patients require frequent clinical reassessment (every 1-2 days) until either a diagnosis is made and definitive therapy instituted, or until subjective and objective clinical improvement is achieved. Prior to initiation of empiric therapy as above, all samples for microbiological testing should have been obtained as outlined in Step 3.

ii) Caution must be exercised in the evaluation and empiric therapy of fever in children, given the higher rate of complicated disease in this age group. Clinicians should have a low threshold for admission and initiation of IV therapy for presumed enteric fever, in which case a parenteral third generation cephalosporin (ceftriaxone or cefotaxime) could be considered. Similarly, use of doxycycline and ciprofloxacin is not routine in children and generally reserved for specific cases in which the benefits of these antibiotics are felt to exceed the potential risks (permanent dental staining, arthropathy). Consultation with a specialist experienced in the treatment of pediatric tropical diseases should be considered.

*3-day regimen for suspected travellers' diarrhea; 7-10-day regimen for suspected enteric fever

$* *$ this will also cover leptospirosis 


\section{Appendix I. Specific tests to rule out common travel-acquired infections that can cause fever}

\begin{tabular}{|c|c|}
\hline Travel-acquired infection & Diagnostic test(s) \\
\hline Malaria & $\begin{array}{l}\text { 1. Thick and thin blood smears } \pm \text { antigen-based dipstick assay; minimum 2-3 times over 24-48 } \\
\text { hours } \\
\text { 2. Blood for malaria polymerase chain reaction (PCR) if smears and/or dipstick negative but } \\
\text { index of suspicion high }\end{array}$ \\
\hline $\begin{array}{l}\text { Acute travellers' } \\
\text { diarrhea / } \\
\text { gastroenteritis } \\
\text { (60-80\% bacterial) }\end{array}$ & $\begin{array}{l}\text { 1. Stool culture for enteropathogens x } 1 \text { (will detect Salmonella, Shigella, Campylobacter, E. coli } \\
\text { O157:H7, and often Yersinia) } \\
\text { 2. Stool for Clostridium difficile toxin } \\
\text { 3. Stool for ova and parasites (O\&P) x } 3 \text { (be aware that not all laboratories screen for all pro- } \\
\text { tozoa, including coccidia, routinely; check with local laboratory for special staining request } \\
\text { requirements) } \\
\text { 4. Amoebic serology } \pm \text { stool Entamoeba histolytica ELISA if bloody stool }\end{array}$ \\
\hline RTI & $\begin{array}{l}\text { 1. Chest x-ray } \\
\text { 2. Nasopharyngeal swab (NP) swab for viral antigen testing or PCR (influenza A/B, respiratory } \\
\text { syncytial virus [RSV], adenovirus, parainfluenza virus 1-3, human metapneumovirus, corona- } \\
\text { virus) } \\
\text { 3. Sputum for culture and susceptibility (C\&S) and acid-fast bacilli (AFB) (as directed by index } \\
\text { of suspicion) } \\
\text { 4. Legionella urine antigen } \\
\text { 5. Epstein-Barr virus (EBV) - EBV monospot unreliable in children } \leq 4 \text { years of age; EBV viral } \\
\text { capsule antigen (VCA) IgM/IgG, EBV nuclear antigen (EBNA) IgM/lgG } \\
\text { 6. (Serology for Q-fever, Histoplasma, Blastomyces, Coccidioides as directed by index of suspicion } \\
\text { and travel exposures; urinary antigen for Histoplasma) }\end{array}$ \\
\hline Dengue & 1. Acute and convalescent sera ( 2 weeks after) for dengue $\operatorname{IgM}$ and $\operatorname{IgG}$ \\
\hline $\begin{array}{l}\text { Enteric fever due to } \\
\text { Salmonella enterica serovar } \\
\text { Typhi or Paratyphi }\end{array}$ & $\begin{array}{l}\text { 1. Blood culture } \times 2 \text { (caution if the patient has received antibiotics as they may have negative } \\
\text { blood cultures) } \\
\text { 2. Stool culture } \\
\text { 3. (Bone marrow aspirate and culture) }\end{array}$ \\
\hline Skin and soft tissue infection & $\begin{array}{l}\text { 1. Clinical diagnosis } \\
\text { 2. Skin swab for methicillin-susceptible and methicillin-resistant Staphylococcus aureus (MSSA } \\
\text { and MRSA) if exudative } \\
\text { 3. If ulcerative, smears for Giemsa-stain, biopsy or aspirate for Leishmania culture or PCR; con- } \\
\text { sider skin swab to rule out ecthyma ulcer due to Staphylococcus or Pseudomonas }\end{array}$ \\
\hline Rickettsioses & $\begin{array}{l}\text { 1. Clinical diagnosis - presence of an eschar is diagnostic (but may not be present) } \\
\text { 2. Acute and convalescent sera for rickettsial serology }\end{array}$ \\
\hline
\end{tabular}




\begin{tabular}{|c|c|}
\hline Acute UTI / STI & $\begin{array}{l}\text { 1. Urinalysis and urine microscopy } \\
\text { 2. Urine culture } \\
\text { 3. Urine and/or endocervical swabs for GC/CT } \\
\text { 4. Swab for viral PCR of genital vesicles } \\
\text { 5. Blood for HIV, HBV, HCV and syphilis serology }\end{array}$ \\
\hline Viral hepatitis & $\begin{array}{ll}\text { 1. } & \text { HAV - HAV IgM, HAV IgG (unless history of previous vaccination) } \\
\text { 2. HBV - HBsAg (surface antigen), HBsAb (surface antibody), HBcAb (core antibody), } \\
\text { HBeAg (e antigen), HBeAb (e antibody); HBV DNA } \\
\text { 3. HCV - HCV total antibody } \\
\text { 4. Hepatitis D virus (HDV) - Anti-HDV antigen; serum HDV RNA reverse transcription } \\
\text { PCR (RT-PCR) } \\
\text { 5. Hepatitis E virus (HEV) - Anti-HEV IgM antibody; blood or stool for HEV PCR } \\
\text { 6. } \\
\text { 7. } \\
\text { 7. Cytomegalovirus (CMV) - IgM/IgG; CMV antigenemia; serum for CMV PCR }\end{array}$ \\
\hline $\begin{array}{l}\text { Other potentially } \\
\text { travel-acquired infections } \\
\text { diagnosed by serology }\end{array}$ & $\begin{array}{l}\text { 1. Viral - Chikungunya, arboviruses } \\
\text { 2. Bacterial - Q-fever, Brucella (can also be cultured from blood or bone marrow), Leptospira } \\
\text { 3. Fungal - Histoplasma, Blastomyces, Coccidioides, Cryptococcus (can detect by serum or CSF or } \\
\text { urinary antigen also) } \\
\text { 4. Parasitic - Strongyloides, Schistosoma, Amoebiasis (can also detect in stool O\&P and by stool } \\
\text { ELISA) }\end{array}$ \\
\hline
\end{tabular}




\section{Appendix II. Additional resources for guidance on fever in the returning traveller}

\section{Committee to Advise on Tropical Medicine and Travel (CATMAT) Statements}

Canadian Recommendations for the Prevention and Treatment of Malaria among International Travellers (2009)

http://www.phac-aspc.gc.ca/publicat/ccdr-rmtc/09vol35/35sl/index-eng.php

Statement On Persistent Diarrhea In The Returned Traveller (2006)

http://www.phac-aspc.gc.ca/publicat/ccdr-rmtc/06vol32/acs-01/index-eng.php

Statement on Travellers and Sexually Transmitted Infections (2006)

http://www.phac-aspc.gc.ca/publicat/ccdr-rmtc/06vol32/acs-05/index-eng.php

\section{Public Health Agency of Canada}

Travel Health Notices website

http://www.phac-aspc.gc.ca/tmp-pmv/pub-eng.php

National Microbiology Laboratory

http://www.nml-lnm.gc.ca/index-eng.htm

Canadian Malarial Network Coordinators (available on the Public Health Agency of Canada's website)

http://www.phac-aspc.gc.ca/tmp-pmv/quinine/index-eng.php

\section{United States - Centers for Disease Control and Prevention}

http://www.cdc.gov/

Viral Special Pathogens Branch, NCID

http://www.cdc.gov/ncidod/dvrd/spb/mnpages/disinfo.htm

VSPB specimen submission info

http://www.cdc.gov/ncidod/dvrd/spb/mnpages/specimen.htm (phone number 404-639-2888)

\section{International}

World Health Organization outbreak site

www.who.int/csr/don/en

Promed

www.promedmail.org 


\section{Articles for further reading}

Johnston V, Stockley JM, Dockrell D, et al. Fever in returned travellers presenting in the United Kingdom:

Recommendations for investigation and initial management. J Infection 2009;59:1-18.

Freedman DO, Weld LH, Kozarsky PE, et al. Spectrum of disease and relationship to place of exposure in ill returned travellers. N Engl J Med 2006;354:119-130.

Boggild AK, Page AV, Keystone JS, Morris AM, Liles WC. Delay in diagnosis: malaria in a returning traveller. CMAJ 2009;180(11):1129-1131. Available at: http://www.cmaj.ca/cgi/content/full/180/11/1129

Rynor B. Dengue fever on the rise at tourist getaways. CMAJ 2010;182(4):E195-196.

Available at: http://www.cmaj.ca/cgi/content/full/182/4/E195

Szakacs TA, McCarthy AE. An all-inclusive vacation. CMAJ 2007;177(1):29-31.

Available at: http://www.cmaj.ca/cgi/content/full/177/1/29

Daneman N, Slinger R. Tache noire. CMAJ 2008;178(7):841.

Available at: http://www.cmaj.ca/cgi/content/full/178/7/841 


\section{Glossary}

\begin{tabular}{|c|c|}
\hline $\mathrm{AFB}$ & Acid fast bacilli \\
\hline BID & Bis in die (twice per day) \\
\hline CATMAT & Committee to Advise on Tropical Medicine and Travel \\
\hline $\mathrm{CDC}$ & Centers for Disease Control and Prevention \\
\hline CMV & Cytomegalovirus \\
\hline CSF & Cerebrospinal fluid \\
\hline C\&S & Culture and susceptibility \\
\hline DNA & Deoxyribonucleic acid \\
\hline DVT & Deep vein thrombosis \\
\hline EBV & Epstein-Barr virus \\
\hline EBNA & Epstein-Barr virus nuclear antigen \\
\hline EBV VCA & Epstein-Barr virus viral capsule antigen \\
\hline ELISA & Enzyme-linked immunosorbent assay \\
\hline GC/CT & Gonorrhea (gonococcus) / Chlamydia trachomatis \\
\hline HAV & Hepatitis A virus \\
\hline $\mathrm{HBcAb}$ & Hepatitis B core antibody \\
\hline HBeAg & Hepatitis B e antigen \\
\hline HBsAb & Hepatitis B surface antibody \\
\hline HBsAg & Hepatitis B surface antigen \\
\hline HBV & Hepatitis B virus \\
\hline $\mathrm{HCV}$ & Hepatitis $C$ virus \\
\hline HDV & Hepatitis D virus \\
\hline HEV & Hepatitis E virus \\
\hline HIV & Human immunodeficiency virus \\
\hline HSV & Herpes simplex virus \\
\hline IBD & Inflammatory bowel disease \\
\hline $\operatorname{IgM}$ & Immunoglobulin $\mathrm{M}$ \\
\hline $\operatorname{Ig} G$ & Immunoglobulin G \\
\hline IV & Intravenous \\
\hline MRSA & Methicillin-resistant Staphylococcus aureus \\
\hline MSSA & Methicillin-sensitive Staphylococcus aureus \\
\hline NP & Nasopharyngeal swab \\
\hline $\mathrm{O \& P}$ & Ova and parasites \\
\hline PCR & Polymerase chain reaction \\
\hline PEP & Post-exposure prophylaxis \\
\hline PHAC & Public Health Agency of Canada \\
\hline po & Per os (by mouth) \\
\hline RNA & Ribonucleic acid \\
\hline RSV & Respiratory syncytial virus \\
\hline RT-PCR & Reverse transcription polymerase chain reaction \\
\hline RTI & Respiratory tract infection \\
\hline STI & Sexually transmitted infection \\
\hline TB & Tuberculosis \\
\hline UTI & Urinary tract infection \\
\hline VFR & Visiting friends and relatives \\
\hline WHO & World Health Organization \\
\hline WNV & West Nile virus \\
\hline
\end{tabular}




\section{References}

1. Committee to Advice on Tropical Medicine and Travel. Canadian Recommendations for the Prevention and Treatment of Malaria Among International Travellers. CCDR 2009;34:1-45.

2. Steffen R, Rickenbach M, Wilhem U, Helminger A, Schar M. Health problems after travel to developing countries. J Infect Dis 1987;156:84-91.

3. Hill D. Health problems in a large cohort of Americans traveling to developing countries. J Travel Med 2000;7:259-266.

4. Wilson M, Weld L, Boggild A et al. Fever in returned travelers: results from the GeoSentinel Surveillance Network. Clin Infect Dis 2007;44:1560-1568.

5. Bottieau E, Clerinx J, Schrooten W et al. Etiology and outcome of fever after a stay in the tropics. Arch Intern Med 2006;166:1642-1648.

6. Doherty J, Grant A, Bryceson A. Fever as the presenting complaint of travellers returning from the tropics. QJM 1995;88:277-281.

7. O'Brien D, Tobin S, Brown G, Torresi J. Fever in returned travelers: review of hospital admissions for a 3-year period. Clin Infect Dis 2001;33:603-609.

8. Antinori S, Galimberti L, Gianelli E et al. Prospective observational study of fever in hospitalized returning travelers and migrants from tropical areas, 1997-2001. J Travel Med 2004;11:135-142.

9. Parola P, Soula G, Gazin P, Foucault C, Delmont J, Brouqui $P$. Fever in travelers returning from tropical areas: prospective observational study of 613 cases hospitalised in Marseilles, France, 1999-2003. Travel Med Infect Dis 2006;4:61-70.
10. West N, Riordan F. Fever in returned travellers: a prospective review of hospital admissions for a 2(1/2) year period. Arch Dis Child 2003;88:432-434.

11. Klein J, Millman G. Prospective, hospital based study of fever in children in the United Kingdom who had recently spent time in the tropics. BMJ 1998;316:1425-1426.

12. Hagmann S, Neugebauer R, Schwartz E et al. Illness in children after international travel: analysis from the GeoSentinel Surveillance Network. Pediatrics 2010;125:e1072-e1080.

13. Freedman DO, Weld LH, Kozarsky PE et al. Spectrum of Disease and Relation to Place of Exposure among Ill Returned Travelers. N Engl J Med 2006;354:119-130.

14. Cooke F, Day M, Wain J, Ward L, Threlfall E. Cases of typhoid fever imported into England, Scotland and Wales (2000-2003). Trans R Soc Trop Med Hyg 2007;101:398-404.

15. Freedeman DO. Malaria prevention in short-term travellers. N Engl J Med 2008;359:603-612.

16. Centers for Disease Control and Prevention. CDC surveillance summaries - malaria surveillance: United States 2004. MMWR 2006;55:23-37.

17. Behrens R, Collins M, Botto B, Heptonstall J. Risk for British travellers of acquiring hepatitis A. BMJ 1995;311:193. 